\title{
Laser-Cooled Ion Crystals
}

\author{
G. Birkl, S. Kassner and H. Walther \\ Max-Planck-Institut für Quantenoptik, Garching bei München, Germany
} The crystal-like structures of laser-cooled ions predicted by molecular dynamics calculations and
recently observed in a miniature quadrupole storage ring have far-reaching implications.

In contrast to neutral atoms, ions are more easily influenced by electric fields because of their charge. Dynamic confinement of charged particles in three dimensions can be achieved using alternating electric fields and an appropriate set of electrodes (a so-called Paul trap after its inventor). Ions in such a trap are confined to a small region in space, thus eliminating transit time broadening. Moreover, the particles can be cooled using a laser beam with a frequency below the resonance frequency. Due to the Doppler effect, an ion is shifted into resonance as it moves in the opposite direction to the light beam; photons are absorbed whereby the particle's linear momentum is reduced by photon recoil. The spontaneously emitted photons have random directions so their momentum transfer is averaged out and a net momentum transfer is only achieved during the excitation process. The residual velocities of the stored ions at the achievable temperatures of a few milli-Kelvins or less are so small that first and second-order Doppler effects both disappear.

As the particles are trapped in an ultrahigh vacuum, there are no collisions which could broaden the resonance line. Trapped, laser-cooled ions therefore offer unique possibilities for ultra-high precision spectroscopy and may provide the basis for new optical and microwave frequency standards (see, e.g., [1] for a survey). Single-ion experiments can in addition be used to demonstrate quantum effects in atom-radiation interaction such as, for example, the antibunching of photons emitted in resonance fluorescence. Owing to the almost constant duration of an absorption-emission cycle, the emitted photons show sub-Poissonian statistics [2] that are not observed with the usual light sources, or even with laser light, where the statistics are Poissonian. Another example is the demonstration of quantum jumps with a single stored ion [3]. It was

Gerhard Birkl received his diploma in physics from the University of Munich in 1990. He is presently working towards his Ph.D. at the MPI für Quantenoptik, Postfach 1513, W-8046 Garching.

Sven Kassner studied physics at the University of Hannover and the Technical University, Munich, completing his diploma in 1990. $\mathrm{He}$ is presently undertaking a Ph.D. at the MPI für Quantenoptik.

Professor Herbert Walther, the 1992 EPS Lecturer, has been with the University of Munich since 1975. He is the Director of the MPI für Quantenoptik and a scientific member of the Max-Planck-Gesellschaft.
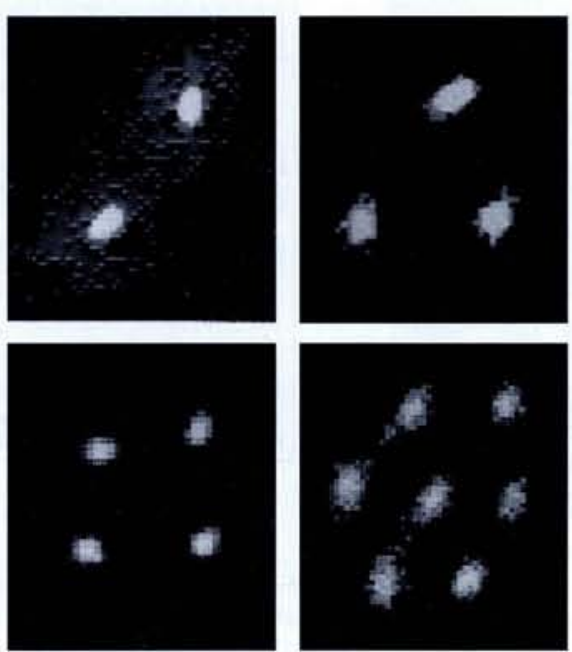

Fig. 1 - Crystals of two, three, four, and seven laser-cooled $\mathrm{Mg}^{+}$ions confined in a Paul trap and observed using resonantly scattered light. The average ion separation is $20 \mu \mathrm{m}$.

possible in these experiments to observe the transition of an ion from an excited state to a lower state so that the lifetime of the ion in a particular upper state could be monitored for each excitation cycle.

Using a Paul trap, we have also been able to study the physics of few-body phase transitions of laser-cooled magnesium ions [4] and the ordered state of the trapped ions could be observed directly by monitoring their resonantly scattered light (Fig. 1). The ordered structures, representing configurations of maximum density for the ions, are determined by the trap potential pushing the ions towards the trap centre, and by the Coulomb repulsion between the ions acting in the opposite direction so that minimum energy configurations are finally adopted

Similar structures are expected in highenergy storage rings for ions when the

Fig. 2 - Experimental set-up of the quadrupole storage ring, including the atomic beam oven and the electron gun. The storage ring contained in an ultra-high vacuum chamber with a pressure below $10^{-8} \mathrm{~Pa}$ consists of four circular electrodes with a diameter of $115 \mathrm{~mm}$ (an enlarged cross-section of the electrode system is shown in the inset). The laser beam for

ion cooling enters the storage volume tangentially. Resonance fluorescence is detected in the radial direction with a photomultiplier tube and an imaging photon detector system. phase space distribution of the ions is reduced by laser cooling [5]. To date, only theoretical results using molecular dynamics (MD) simulations [6] of ordered ion beams in these storage rings have been reported. The first experimental steps towards crystalline beams have been the cooling of ${ }^{7} \mathrm{Li}^{+}$to the Kelvin and milli-Kelvin regimes [7].

\section{Quadrupole Storage Ring}

It is possible to observe crystalline structures of ions in a low-energy quadrupole storage ring [8] as they are much easier to realise than for high-energy storage rings, squeezing and focussing sections, Coulomb explosions in the drift sections and shearing forces in the bending sections. We have investigated these structures in a miniature quadrupole storage ring for a wide variety of experimental conditions, and the results confirm theoretical predictions.

The small storage ring also has interesting possibilities as a trap for high-resolution spectroscopy. Ions sitting along the axis of the quadrupole field do not show the phenomenon of micromotion in the same way as a single ion stored at the centre of a Paul trap, where ions away from the trap centre are affected by the alternating voltage applied to the trap electrodes and therefore move periodically with the frequency of the trap voltage. The ion storage ring thus has the advantage that many ions can be investigated under Doppler-free conditions. Moreover, an increase in the number of ions stored improves the signal-to-noise ratio.

\section{Experimental}

The miniature quadrupole storage ring is shown in Fig. 2. The set-up is contained in an ultra-high vacuum chamber with a preswhere the ions are subjected to periodic 

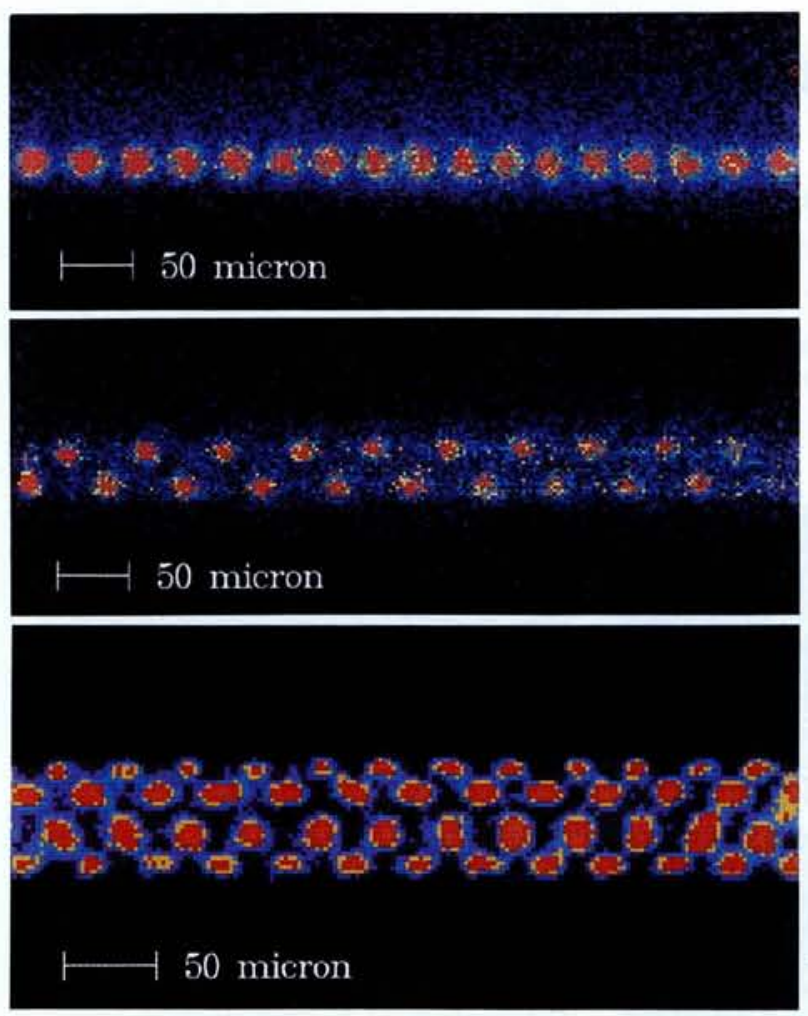

Fig. 3 - Crystalline structures of laser-cooled ${ }^{24} \mathrm{Mg}+$ ions in the quadrupole storage ring. At a low ion density $(\lambda=0.29)$ the ions form a string along the field axis (a, upper). Increasing the ion density transforms the configuration to a zigzag ( $b$, middle; $\lambda=$ 0.92). At still higher ion densities, the ions form ordered helical structures on the surface of a cylinder, e.g., three interwoven helices at $\lambda=2.6$ (c, lower). As the fluorescent light is projected onto the plane of observation in this case the inner spots are each created by two ions seated on opposite sides of the cylindrical surface, resulting in a single, bright spot.

sure below $10^{-8} \mathrm{~Pa}$ maintained with an ion pump. The storage device consists of pairs of inner and outer rings held together by ceramic supports. Gold-coated stainless steel electrodes with a distance of $5 \mathrm{~mm}$ between opposing electrodes define a toroidal trap region with a diameter of $115 \mathrm{~mm}$. Those parts of the electrodes which face the trap region have a circular cross-section of $2.9 \mathrm{~mm}$ in radius. Trapping is accomplished by applying a RF voltage of $6.56 \mathrm{MHz}$ and an amplitude of a few hundred volts, resulting in a harmonic potential well with a depth $\psi_{\mathrm{o}}$ of several electron volts in the plane transverse to the electrodes. Ions can move freely in the direction along the field axis. There is a $2 \mathrm{~mm}$ gap between the electrodes, affording space for atomic, electron and laser beams to enter the trap region.

We used for our experiments magnesium ions produced by electron bombardment of a thermal atomic beam. A contact potential (the voltage difference created by the direct contact of two metals) is produced by depositing stray magnesium atoms onto the electrodes in the region where the atomic beam passes through the quadrupole. The potential of the coated trap section is roughly $1 \mathrm{eV}$ above the remainder of the ring.

The trapped particles oscillate in the harmonic potential well and their motion can be

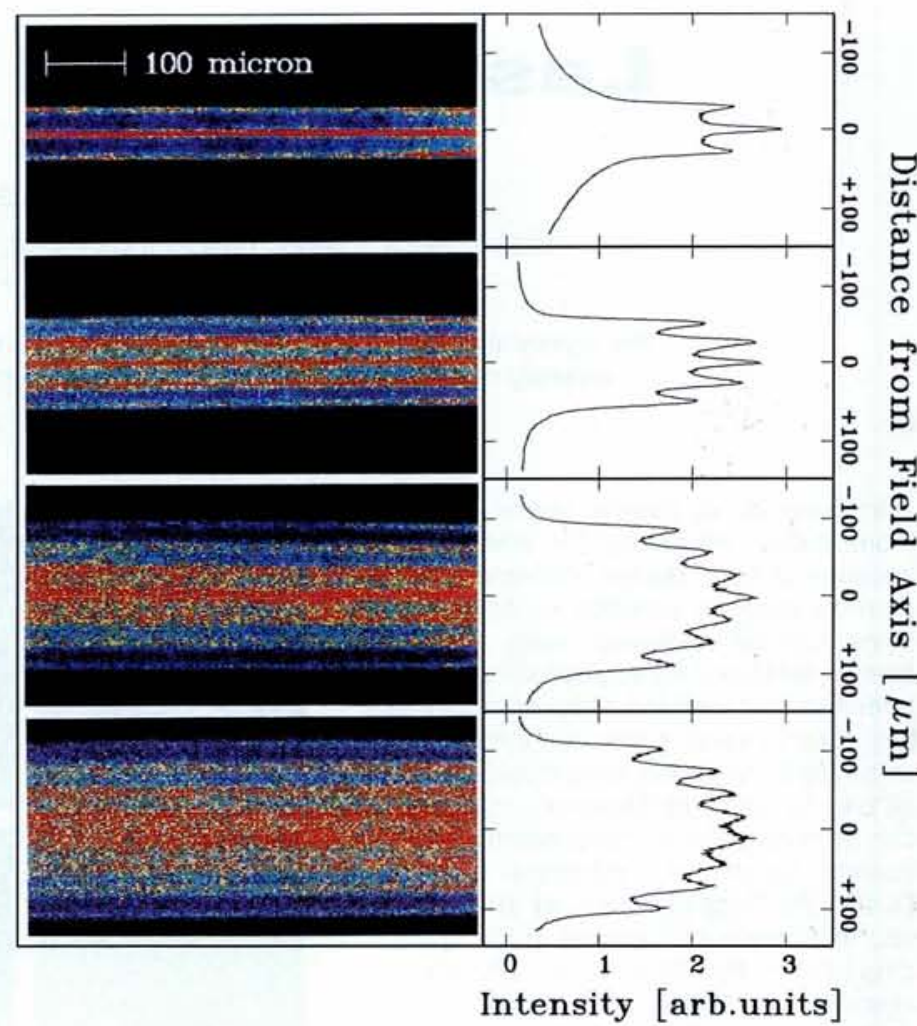

Fig. 4 - Images and intensity profiles of (from the top): one shell plus string $(a ; \lambda \approx 4.3)$, two shells plus string ( $b ; \lambda \approx 12.2)$, three shells plus string $(c ; \lambda \approx 26)$, and four shells $(d ; \lambda \approx 34)$. There are up to $\approx 8 \times 10^{5}$ ions stored in the ring for the four-shell structure. damped by laser cooling using the $3^{2} \mathrm{~S}_{1 / 2}$ ${ }^{2} \mathrm{P}_{3 / 2}$ transition of ${ }^{24} \mathrm{Mg}^{+}$at $280 \mathrm{~nm}$. If the laser frequency is tuned into resonance from below, the velocities of the ions are changed so that the ions finally end up with a bunched (cooled) velocity distribution, moving in the direction of the laser beam. As the ions can travel freely along the field axis, two opposing laser beams are usually needed to cool all stored ions to rest. We achieve efficient cooling with a single beam because of the contact potential: lasercooled ions with a kinetic energy below $1 \mathrm{eV}$ cannot pass the potential barrier. They oscillate parallel to the field axis and periodically come into resonance with the laser light. Fast-moving ions are first cooled sympathetically via Coulomb collisions with slower particles until they are unable to cross the barrier, which then compensates for the radiation pressure. Cooling leads to a spectroscopically observable phase transition and to ordered structures of the laser cooled ions [8]. By imaging the fluorescence of the ions onto a position-sensitive photon detector we can directly obtain images of the equilibrium configurations, with the ions being resolved individually.

\section{Ordered structures}

In the MD calculations, a cylindrically symmetric, static harmonic potential is assumed to describe the confining field. Each particle is subjected to the Coulomb forces of all other particles and to the confining field. The classical equations of motion are integrated for a system of several thousand particles, starting with random positions and velocities, to give the time evolution of the system. Cooling of the stored particles is simulated by scaling down the resulting velocities of the stored particles at defined instants of the integration process. After sufficient cooling, ordered structures such as strings, zigzags, shells, and multiple shells should arise owing to the confining field's harmonic potential [6]. Our experiments are well-suited to checking these predictions. To compare the experimental results with theory, we can use the normalised "linear particle density" $\lambda$ which is given by the ion density multiplied by the ratio of Coloumb repulsion and confining force of the trap [6]. Low $\lambda$ values correspond to a deep potential well or a small number of ions, resulting in an equilibrium structure closely confined to the field axis comprising a string of ions (Fig. $3 a)$. This is the micromotion-free configuration discussed above, and the analogue of the single stored ion in a Paul trap, as in both cases the ions sit in the potential minimum and show no micromotion. For higher values of $\lambda$, the structures extend more and more into the off-axis region, giving rise to (in the order of increasing $\lambda$ ) a plane zigzag structure (Fig. 3b) and cylindrical structures with the ions forming helices on cylindrical surfaces. The structure in Fig. $3 \mathrm{c}$ consists of three interwoven helices with six ions per pitch. The string and the zigzag have also been observed with laser-cooled $\mathrm{Hg}^{+}$ions in a linear trap [9].

Increasing further the number of ions leads to structures with smaller spacings between the ions where we cannot optically resolve individual ions any more. Images of 


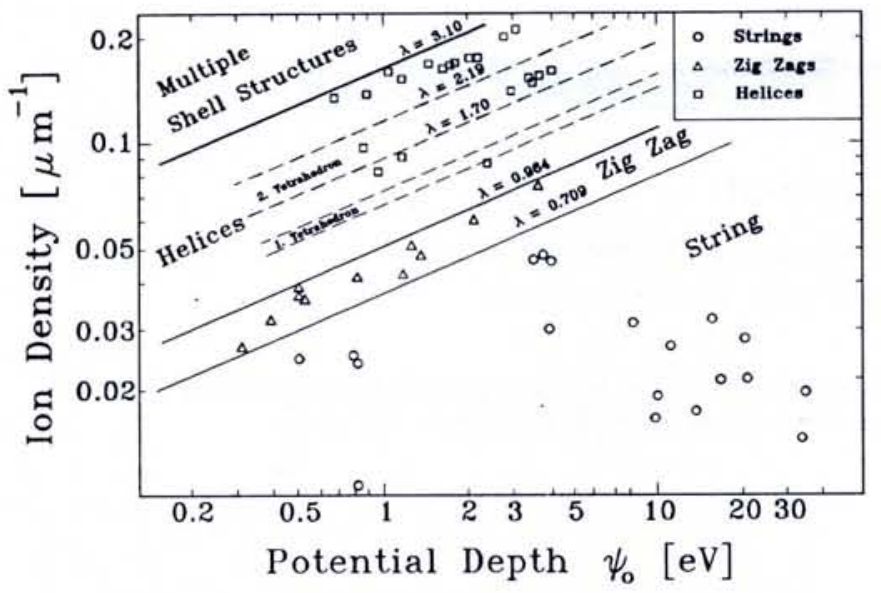

Fig. 5 - Summary of the experimental results. (a, left) Individual ions resolved, where the observed structures are characterised by the ion density per unit length and the depth of the potential well $\psi_{0}$. These two parameters can be combined to give the normalised linear particle density $\lambda$ which fully determines the ion configuration. The straight lines show critical $\lambda$ values separating the regions corresponding to the various theoretically expected structures. The observed configurations are labelled with different symbols for each structure. (b, right) Individual ions unresolved, where the observed shell structures with up to four shells plus string are characterised by their radius $\rho$ and the potential depth $\psi_{0}$. The various observed structures are again separated by lines of theoretically determined critical $\lambda$.

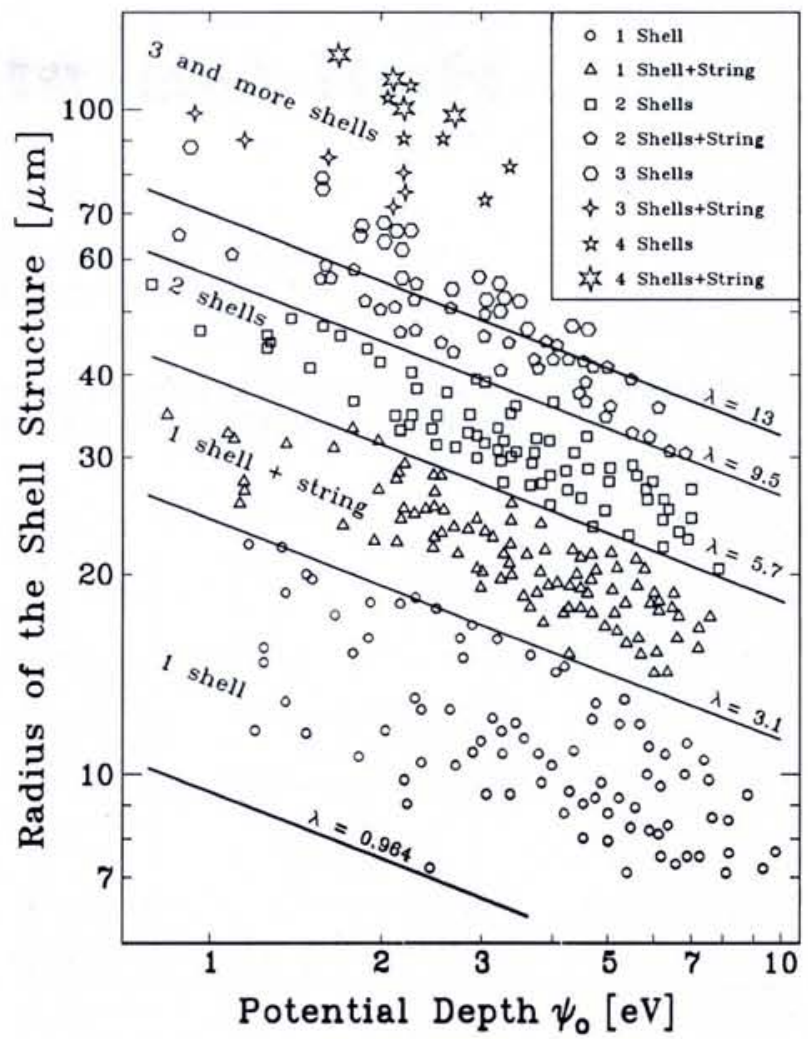

available in the ring will lead to a high signal-to-noise ratio. Finally, cooled ions in the ring represent a quantum object of macroscopic dimensions (a Wigner crystal).

Second, with the experimental confirmation that the ordered structures expected in high-energy ion storage rings can indeed be formed, a completely new era of accelerator physics will emerge if it is possible to reproduce such Coulomb crystals in these rings. The enhanced luminosity of the corresponding beams would allow studies of ionic reactions whose cross-sections are too small for investigations in existing accelerators.

[1] Frequency Standards and Metrology, Ed.: A. De Marchi (Springer, Berlin)1989.

[2] Diedrich F. and Walther H., Phys. Rev. Lett. 58 (1987) 203.

[3] E.g., Nagourney W., Sandberg J. and Dehmelt H., Phys. Rev. Lett. 56 (1986) 2797.

[4] Diedrich F. et al., Phys. Rev. Lett. 59 (1987) 2931; Blümel R. et al., Nature 334 (1988) 309; Blümel R. et al., Phys. Rev. A 40 (1989) 808.

[5] Proc. Workshop on Crystalline Ion Beams, Eds.: R.W. Hasse, I. Hoffmann and D. Liesen (GSI, Darmstadt) 1989

[6] Hasse R.W. and Schiffer J.P., Ann. Physics 203 (1990) 419; Rahman A. and Schiffer J.P., Phys. Rev. Lett. 57 (1986) 1133.

[7] Schröder S. et al., Phys. Rev. Lett. 64 (1990) 2901; Hangst J.S. et al., Phys. Rev. Lett. 67 (1991) 1238.

[8] Waki I. et al., Phys. Rev. Lett. 68 (1992) 2007; Birkl G., Kassner S. and Walther H., Nature 357 (1992) 310

[9] Raizen M.G. et al., J. Mod. Opt. 39 (1992) 233.

[10] Gilbert S.L., Bollinger J.J. and Wineland D.J., Phys. Rev. Lett. 60 (1988) 2022.

[11] Dalibard J. and Cohen-Tannoudji C., J. Opt. Soc. Am. B 6 (1989) 2023. 